\title{
Socio-demographic, medical and social-cognitive correlates of physical activity behavior among older adults (45-70 years): a cross-sectional study
}

Ilse Mesters ${ }^{1 *}$, Stefanie Wahl ${ }^{1}$ and Hilde M Van Keulen ${ }^{2}$

\begin{abstract}
Background: Present study aimed to identify socio-demographic, medical and social-cognitive correlates of physical activity among Dutch older individuals.

Methods: A systematic random sample of 2,568 Dutch participants aged 45-70 years filled out the validated modified Community Healthy Activities Model Program for Seniors (CHAMPS) questionnaire on physical activity. Socio-demographic and social-cognitive correlates were measured with validated instruments; medical correlates were checked by a general practitioner. The study had a cross-sectional design and the data collection ran from March 2005 until August 2006. Linear regression analyses were conducted to identify correlates of PA. We separated the findings for men from those for women to explore potential gender-specific associations.

Results: Being female, living in North Limburg or North-Brabant, having a higher educational level, a higher perceived behavioral control, more knowledge about PA advantages, a stronger habitual PA behavior, having more action plans and a stronger intention to engage in PA were significantly associated with higher PA levels. Being older, being a smoker, having a higher body mass index (BMI), having a paid job, observing others being physically active and overestimating one's PA level were associated with being less physically active. Socio-demographic and medical correlates significantly explained $20 \%$ of the variance of PA behavior while social-cognitive correlates as attitude explained an additional $4 \%$ and intention together with actual control explained another $1 \%$ of the variance of PA behavior.

Conclusion: There may be stable individual differences that influence PA in view of the fact that several socio-demographic and medical factors were not completely mediated by the socio-cognitive factors. The current study may help to focus PA interventions for individuals aged 45-70 years on influential socio-demographic, medical and social-cognitive correlates. Physical activity was significantly associated with age, gender, education, BMI, work situation, region of residence, smoking, awareness, advantages, descriptive norm, perceived behavioral control, habit, action plans and intention.
\end{abstract}

Keywords: Physical activity, Prevention, Cardiovascular disease, Intervention, Public health

\section{Background}

Physical Inactivity is considered to be an important risk factor for cardiovascular diseases (CVDs), which are the leading cause of morbidity and mortality worldwide [1]. Several reviews showed that physical activity $(\mathrm{PA})$ may have a preventive effect on CVD mortality and morbidity [2-4]. Other

\footnotetext{
* Correspondence: ilse.mesters@maastrichtuniversity.nl

${ }^{1}$ School for Public Health and Primary Care (Caphri), Department of Epidemiology, Maastricht University, PO Box 616, Maastricht 6200 MD, The Netherlands

Full list of author information is available at the end of the article
}

benefits of PA are prevention of CVD risk factors such as diabetes type II, hypertension, anxiety and depression [5,6].

The Dutch PA guideline is based on international guidelines [5,7] and recommends adults to be physically active with a moderate intensity for 30 minutes 5 times a week. In $201159 \%$ of the Dutch population (18 years and older) and $70 \%$ of the people aged $55-75$ years met the national PA guideline [8] For the development of an intervention to promote $\mathrm{PA}$, knowledge about relevant and changeable determinants of PA is needed [9]. To understand how these determinants are mutually related, 
it has been recommended that determinant studies preceding behavioral change interventions should be grounded in theory [10]. Developers of health behavior interventions have often drawn on the Theory of Reasoned Action (TRA), respectively the Theory of Planned Behavior (TPB) [11], which explain the process through which people change their behavior [11,12]. According to these theories, the intention state for engaging in PA predicts future PA behavior [11]. The transition from intention to behavior is influenced by actual control (environmental factors, barriers, abilities, skills and action plans). The intention state, in turn, is determined by social-cognitive factors such as attitude, perceived norms and perceived behavioral control. Attitude refers to the evaluation of behavior in terms of cognitive and emotional advantages and disadvantages. Perceived norms include for example recognizing other individuals performing the type of behavior (descriptive norms). In this study, beliefs about whether specific social referents approve or disapprove engaging in PA (injunctive norms) was replaced by the construct social support, since the latter was described as having a much stronger influence on behavior [13]. Perceived behavioral control is the perceived ability to perform a health behavior. The social-cognitive factors are influenced by factors such as individual (e.g. personality, habit, and awareness), social-demographic (e.g. education, age) medical (e.g. having hypertension, diabetes, high BMI) and information factors (e.g. exposure to media). For instance, when people are in the habit of being physically active the predictive power of the social cognitive variables may be attenuated.

While several studies [14-16] have been conducted to investigate determinants of PA behavior, few $[17,18]$ of them have focused on older adults (aged 45-70). However, several reasons exist to concentrate on older adults as target group for improving PA levels. Not only is $36.9 \%$ of the European population older than 50 years [19], but also this percentage will increase due to the aging of the population. Moreover, regular PA in older individuals contributes to an increase in longevity, a reduction of pain from arthritis, a decrease of risk to falls and fractures, and an increase in the ability to maintain functional independence [6]. Thus, stimulating PA among this large and growing group is of great relevance to minimize the public health burden.

The first aim of the study was to explore which sociodemographic, medical and social-cognitive factors have the strongest link with self-reported PA behavior in a population-based sample of adults aged 45-70 years living in the Netherlands.

Moreover, few Dutch studies have explored whether correlates of PA differ for middle aged men and women. It is expected that the associations may vary between the two [20].

Therefore, the second aim was to explore PA patterns and associated variables for men and women separately.

\section{Method}

\section{Participants}

A systematic random sample of patients was drawn from 23 general practices in the regions Limburg (19 practices) and North-Brabant (4 practices). These practices were connected to the RNH (Registration Network Family Practices) data base which allowed computer generated random selection of patients [21]. Selection variables included age (45-70 years), and $\pm 50 \%$ had general practitioner (GP) diagnosed hypertension based on the International Classification of Primary Care (ICPS code K86 or K87) $[22,23]$ and about $50 \%$ had no hypertension. Additionally, selected persons should not be involved in other studies according to the general practice database and only one person per address was allowed to enter the study. Of 6,420 computer-selected participants 875 were excluded due to further selection criteria by the GP (e.g. unknown address, physically unable to comply to healthy lifestyle, not able to speak/read Dutch, life-threatening or malignant disorders). Invitations for participating in the study were mailed to 5,545 individuals and the 2,881 individuals who consented to participate received a printed questionnaire. Finally, 2,568 participants returned the printed questionnaire. For a full overview about the selection and enrollment of the Vitalum participants, see Van Keulen and colleagues [24]. The recruitment was done in waves and lasted from March 2005 until August 2006. The study was approved by the medical ethics committee of Maastricht University and Maastricht Medical Hospital (azM).

\section{Design}

Baseline data of the Vitalum study were used for this cross-sectional study [25]. The Vitalum study simultaneously evaluated the efficacy of tailored print communication and telephone motivational interviewing, and their combined use for multiple health behaviors in individuals with and without hypertension and of diverse education levels. With the inclusion of participants with hypertension (systolic blood pressure $>140 \mathrm{mmHg}$ ), it was investigated whether the existence of a physician diagnosed medical CVD risk factor had an influence on PA behavior.

\section{Measurements}

The TRA model indicates that other variables than intention and it antecedents (social-cognitive variables), can be associated with behavior. However, the influences of such background variables are expected to be indirect.

\section{Back ground variables}

Age, gender, weight and height (for body mass index (BMI) calculation), highest completed education, work situation, marital status, family history of cardiovascular diseases [26], diabetes, living situation and native country were measured with a written questionnaire. Information 
about gender, hypertension status, and region of residence were provided by the GPs after participants agreed to take part in the study. The variable awareness was measured with two questions (for example Do you rate your PA level as low or high?; $1=$ low, $5=$ high [14] and the answers were compared to the reported PA levels in the questionnaire [26,27]. Accordingly, participants were categorized in two groups: 1. Overestimators who did not meet the guidelines but rated their PA level as high; 2. Underestimators who met the Dutch guideline but rated their PA level as low, or realists who estimated their PA level correctly. Habit was assessed by the frequency of engaging in physical activity $(\alpha .88)$ [15]; To what extent do you agree with the following statements: for example "Being physically active is something I regularly do" ( $1=$ completely disagree, $5=$ completely agree). The questionnaire further included one item for stress, two items for smoking behavior and two items for alcohol consumption. Stress was operationalized by asking whether the participants experience a lot of stress [28]. Smoking was assessed by asking the frequency and quantity of tobacco use [29]. Alcohol intake was measured by questions about the frequency and quantity of alcohol use, resulting in a drinking score below or above the national recommendation [30]. The alcohol national consumption guideline specifies a consumption of less than three glasses a day for men and less than two glasses a day for women [31]. As PA patterns may vary by season [32], the variable season was computed using the date on which participants returned the questionnaire.

\section{Physical activity behavior}

PA was measured with the modified version of the Community Healthy Activities Model Program for Seniors (CHAMPS) questionnaire [33]. The original measure included 41 items [34], the modified questionnaire included 28 items about the frequency (times per week) and duration of physical activities (classified using six categories ranging from „less than $1 \mathrm{~h} \cdot \mathrm{wk}^{-1}$ ”, $1-2.5 \mathrm{~h}$. $\mathrm{wk}^{-1}, 3-4.5 \mathrm{~h} \cdot \mathrm{wk}^{-1}, 5-6.5 \mathrm{~h} \cdot \mathrm{wk}^{-1}, 7-8.5 \mathrm{~h} \cdot \mathrm{wk}^{-1}$ and "9 or more $h \cdot w^{-1 "}$ "). Included activities were walking leisurely/fast or briskly, cycling leisurely/fast or briskly, doing light/heavy gardening, doing light/heavy housekeeping, jogging or running, swimming, playing tennis or badminton, playing team sport indoors or outdoors, doing light exercises to maintain physical condition (stretching, flexibility training) and doing heavy exercises (fitness, strength training). Metabolic equivalents (METs) were determined for each activity on the basis of the compendium of PA by Ainsworth et al. [35].

MET levels were used as cut-offs to calculate the total number of weekly PA hours with at least a moderate intensity. Only activities with at least three METs counted as moderately intense activity for all participants [36]. Participants were classified as adhering to the PA guideline if they were physically active with at least moderate intensity for at least 2.5 hours a week. The number of weekly hours of at least moderate intensive activities was used as primary outcome.

The CHAMPS has been validated [33,37,38]. The reproducibility of the CHAMPS was shown to be good. e.g. [39].

\section{Social-cognitive factors}

Questions about the following variables were included: attitude (advantages and disadvantages), perceived norms (descriptive norm and perceived social support), perceived behavioral control, habit strength, awareness, action plans and intention in form of stages of change (Transtheoretical Model) [40]. Factor analyses with principle axis factoring and promax rotation resulted in two underlying factors for attitude (advantages and disadvantages) and perceived norms (descriptive norm and social support). Habit and perceived behavioral control had one underlying factor. Sum scores calculated by summing up the items of the scale for the factors (advantages, disadvantages, perceived social support, descriptive norm, perceived behavioral control), were used in the data analysis. The social-cognitive concepts from the reasoned action model were operationalized for the current study according to the suggestions by diverse authors (Table 1 ).

\section{Statistical analyses}

Statistical analyses were performed using SPSS 18.0. Statistical significance was assumed for $p$-values $<.05$. Descriptive statistics were performed to describe the sample. Information about missing values, outliers and data checking can be found elsewhere [24,25]. Cronbach's alphas were used to evaluate the internal consistency of the socialcognitive scales. Hours per week moderately physically active acted as dependent variable and required square root transformation to achieve a normal distribution of the residuals. Linear regression was done to determine significant correlates of PA. For the linear regression (sequential multiple regression) the Enter method was used as it is a common procedure for model-based analyses e.g. [41].

To identify the unique contribution of background factors, social-cognitive factors, intention and actual control from the Theory of Reasoned Action, these factors were entered into the regression model in three steps. In the first step, background factors (e.g. age, hypertension, awareness) were entered followed by social-cognitive factors (attitude, perceived norms and perceived behavioral control) in step 2, and intention as well as action plans in step 3. During each step non-significant variables were manually deleted one by one and only significant variables stayed in the model. The same procedure was conducted for investigating gender-specific factors using select cases. 
Table 1 Description and operationalization of social-cognitive variables

\begin{tabular}{|c|c|c|c|c|}
\hline Concepts & \# Items & Example of item question (answer option) & a & Reference \\
\hline Advantages (attitude) & 13 & $\begin{array}{l}\text { Being physically active on at least } 5 \text { days a week for } 30 \text { minutes, } \\
\text { improves my condition ( } 1=\text { completely disagree; } 5=\text { completely agree) }\end{array}$ & 0.86 & {$[16]$} \\
\hline Disadvantages (attitude) & 11 & $\begin{array}{l}\text { Being physically active on at least } 5 \text { days a week for } 30 \text { minutes, } \\
\text { is very time consuming ( } 1=\text { completely disagree; } 5=\text { completely agree) }\end{array}$ & 0.81 & [16] \\
\hline Social support (perceived norm) & 5 & $\begin{array}{l}\text { Do important others (partner, family, friends, doctor or media) } \\
\text { encourage you to be physically active according to the guidelines } \\
\text { ( } 1=\text { completely disagree, } 5=\text { completely agree) }\end{array}$ & 0.81 & {$[16]$} \\
\hline Descriptive norm (perceived norm) & 3 & $\begin{array}{l}\text { Do important others (partner, family, friends) meet the physically } \\
\text { activity norm (physically active for } 30 \text { minutes on at least } 5 \\
\text { days a week) ( } 1=\text { completely disagree, } 5=\text { completely agree) }\end{array}$ & 0.76 & {$[16]$} \\
\hline Perceived behavioral control & 11 & $\begin{array}{l}\text { To what extent would you be able to be physically active on } \\
\text { at least } 5 \text { days a week for } 30 \text { minutes, when you are tired? } \\
\text { ( } 1 \text { = completely unable; } 5=\text { completely able) }\end{array}$ & 0.92 & {$[17]$} \\
\hline Action plan (actual control) & 6 & $\begin{array}{l}\text { If you want to improve your PA level what would you do? } \\
\text { "go for a brisk walk daily" ( } 0=\text { no, } 1=\text { yes })\end{array}$ & - & {$[18]$} \\
\hline Stages of change/intention & 1 & $\begin{array}{l}\text { Which statement fits you best? Ranging from } 1==^{\prime \prime} \text { have no plans } \\
\text { to be physically active on at least } 5 \text { days for } 30 \text { minutes a day } \\
\text { (no motivation) to } 6=" \text { "I have been physically active on at least } \\
5 \text { days a week for } 30 \text { minutes for longer than } 6 \text { months" (maintainer). }\end{array}$ & - & {$[19]$} \\
\hline
\end{tabular}

\section{Results}

Of the 5,545 invited people 4,379 responded; $45 \%(\mathrm{n}=$ 2,881 ) provided informed consent. Reasons for refusal included merely "no interest"or "no time". The questionnaire was returned by 2,568 people ( $89 \%$ of the consenters). Questionnaires were checked at moment of reception for missing data and if present participants were contacted to complete the questionnaire. Sequential regression was employed to determine which background variables and social-cognitive factors were associated with self-reported PA behavior in older adults. Table 2 presents the background and social-cognitive factors of the sample.

\section{Aim 1: Examining associated variables of $\mathrm{PA}$}

Table 3 shows the findings on physical activity as measured with the CHAMPS. Relatively prevalent activities, as walking leisurely and light housekeeping, were performed to some extent by about $80 \%$ of the respondents. For walking at a higher intensity this percentage was halved. Heavy housekeeping was included in the behavior of almost $67 \%$ of the respondents. Cycling leisurely seemed to be an option for $56 \%$ of the respondents and again this number was halved (28\%) when the cycling intensity went up. Doing light or heavy gardening was mentioned by respectively $60 \%$ and $44 \%$ of the respondents. Only a few people reported to go jogging/running (9\%), swimming (11\%), playing (table) tennis/badminton (9\%), and playing a team sport indoors or outdoors (6\%).

In step one of the linear regression analysis, background factors significantly explained $20 \%$ of the variance of PA behavior $\left(\mathrm{F}(10,2457)=61.86, p<.000, \mathrm{R}=.45, \mathrm{R}^{2}=.20\right)$. When controlling for background factors, social-cognitive factors additionally explained $4 \%$ of the variance of PA behavior $\left(\mathrm{F}(13,2397)=57.48, p<.000, \mathrm{R}=.49, \mathrm{R}^{2}=.24\right.$, $\left.\Delta R^{2}=.04\right)$. Furthermore, intention and actual control explained $1 \%$ of the variance of PA behavior in the third step, when controlling for the preceding factors. Age, gender, education, BMI, work situation, region of residence, smoking, awareness, advantages, descriptive norms, perceived behavioral control, habit, action plans and intention remained in the final model as significant correlates of PA behavior (see Table 4). In total, these factors explained $25 \%$ $\left(\mathrm{F}(15,2390)=53.45, p<.000, \mathrm{R}=.50, \mathrm{R}^{2}=.25, \Delta \mathrm{R}^{2}=.01\right)$ of the variance in PA.

\section{In sum}

\section{Background factors}

The results suggest that participants who overestimated their PA level, who were older, who smoked, who had a higher BMI and had paid work were less physically active. In addition, participants with stronger habitual PA behavior, with higher education levels, who were female, and who lived in North Limburg or North-Brabant had higher PA levels.

\section{Social-cognitive factors}

The results indicate that participants who perceived more advantages and had high perceived behavioral control were more physically active. Participants who perceived physically active role models were less physically active.

\section{Intention}

Participants, who formed more action plans and who had a stronger intention were more physically active. 
Table 2 Socio-demographic characteristics, medical characteristics and social-cognitive factors of participants in the study ( $\mathrm{N}=\mathbf{2 5 6 8})$

Variables $\%$ or mean; SD; range
Background factors

\section{Gender}

$$
\% \text { Female }
$$

Age (mean; SD; range)

Native country

$\%$ The Netherlands

\section{7}

$57.4 ; 7.1 ; 44-70$

Educational level*

$$
\begin{aligned}
& \text { \% Low } \\
& \text { \% Intermediate } \\
& \text { \% High }
\end{aligned}
$$

Living situation

$$
\% \text { Together }
$$

Work situation

$$
\% \text { Paid work }
$$

Marital status

$$
\begin{aligned}
& \% \text { Married/in a relationship } \\
& \% \text { Single/divorced/widowed }
\end{aligned}
$$

Region of residence

$\%$ South Limburg

\% North Limburg/North-Brabant

Hypertension

$$
\% \text { Hypertensive }
$$

Body mass index (mean; SD; range)

Diabetes

$\%$ Diabetes

CVD family history

$\%$ One family member with CVD

Perceived stress level

$$
\begin{aligned}
& \% \text { Less than normal } \\
& \% \text { Normal } \\
& \% \text { High }
\end{aligned}
$$

CVD family history

$\%$ One family member with CVD

Smoking behavior

\% Smoker

Alcohol consumption**

Glasses/day (mean; SD; range)

$\%$ Not meeting guidelines

$$
\text { Season }
$$

$$
\begin{aligned}
& \text { \% Spring } \\
& \text { \% Summer } \\
& \text { \% Autumn } \\
& \text { \% Winter }
\end{aligned}
$$

Table 2 Socio-demographic characteristics, medical characteristics and social-cognitive factors of participants in the study ( $N=2568$ ) (Continued)

Physical activity
CHAMPS: hours/week moderately

CHAMPS: $\% \geq 2.5$ hours/week

moderately physically active

Gender specific

$$
\begin{aligned}
& \text { Men (mean; SD; range) } \\
& \text { Women (mean; SD; range) }
\end{aligned}
$$

$5.8 ; 4.8 ; 0-26.5$

$6.8 ; 4.9 ; 0-26.6$

\section{Social-cognitive factors}

Awareness

$$
\begin{aligned}
& \text { \% Overestimator } \\
& \% \text { Underestimator/realists }
\end{aligned}
$$

60.0

39.6

Disadvantages (attitude)

Advantages (attitude)

Social support

Descriptive norm

Perceived behavioral control

Habit

Action plan physically active (mean; SD; range)

Aim 2: Examining whether the variables associated with PA vary by gender Women

Among women, background factors explained 18\% ( $\mathrm{F}(6$, $\left.1133)=41.58, p<.000, \mathrm{R}=.425, \mathrm{R}^{2}=.18\right)$ of the variance of PA behavior. Social-cognitive factors additionally explained $5 \%$ of the variance in PA behavior in step $2(\mathrm{~F}(9$, $\left.1063)=35.53, p<, 000, \mathrm{R}=.48, \mathrm{R}^{2}=.23, \Delta \mathrm{R}^{2}=.05\right)$, and intention and actual control accounted for an additional $1 \%$ of the explained variance in PA behavior in step 3 (F $\left.(9,1104)=38.67, p<.000, \mathrm{R}=.49, \mathrm{R}^{2}=.24, \Delta \mathrm{R}^{2}=.01\right)$.

\section{Men}

Among men background factors explained $22 \%$ ( $\mathrm{F}(10$, $\left.1310)=36.75, p<.000, \mathrm{R}=.47, \mathrm{R}^{2}=.22\right)$ of the variance of PA behavior. Social-cognitive factors additionally explained $3 \%$ of variance in PA behavior in step $2(\mathrm{~F}(11$, $\left.1292)=38.73, p<, 000, \mathrm{R}=.50, \mathrm{R}^{2}=.25, \Delta \mathrm{R}^{2}=.03\right)$. Intention and actual control did not explain additional variance in PA behavior in step $3(\mathrm{~F}(12,1287)=36.36, p<.000, \mathrm{R}=.50$, $\left.\mathrm{R}^{2}=.25, \Delta \mathrm{R}^{2}=.00\right)$.

The following factors varied by gender: Women who were older were less physically active. In contrast, women who made more action plans were more physically active. Men with a higher education level were more physically active. On the contrary, men who drank alcohol above the norm, who smoked, and who were married or living 
Table 3 Champs data

\begin{tabular}{|c|c|c|c|c|c|c|c|c|c|}
\hline \multirow{4}{*}{$\begin{array}{l}\text { How many times per week did you do } \\
\text { underlying activities }\end{array}$} & \multirow{4}{*}{ MET } & \multirow{4}{*}{$\begin{array}{c}\text { Amount } \\
\text { per week } \\
\text { M (sd) }\end{array}$} & \multicolumn{7}{|c|}{ How many hours on average } \\
\hline & & & 0 & $<1$ & $1-2.5$ & $3-4.5$ & $5-6.5$ & $7-8.5$ & $>9$ \\
\hline & & & $\%$ & $\%$ & $\%$ & $\%$ & $\%$ & $\%$ & $\%$ \\
\hline & & & $\mathbf{n}$ & $\mathbf{n}$ & $\mathbf{n}$ & $\mathbf{n}$ & $\mathbf{n}$ & $\mathbf{n}$ & $\mathrm{n}$ \\
\hline \multirow[t]{2}{*}{ Walking leisurely } & 2.5 & $1.98(2.49)$ & 20.2 & 28.7 & 28.5 & 9.8 & 4.5 & 3.7 & 4.2 \\
\hline & & & 518 & 737 & 732 & 252 & 115 & 96 & 109 \\
\hline \multirow[t]{2}{*}{ Walking fast or briskly } & 4 & $.96(1.82)$ & 59.9 & 12.0 & 16.3 & 6.8 & 2.3 & 1.4 & 1.2 \\
\hline & & & 1539 & 308 & 418 & 174 & 60 & 35 & 32 \\
\hline \multirow[t]{2}{*}{ Cycling leisurely (<10 mph) } & 4 & $1.05(1.64)$ & 43.8 & 23.4 & 21.5 & 7.4 & 2.1 & .9 & .8 \\
\hline & & & 1125 & 600 & 551 & 191 & 54 & 24 & 21 \\
\hline \multirow[t]{2}{*}{ Cycling fast or briskly (10-12 mph) } & 6 & $.70(1.66)$ & 72.4 & 8.3 & 9.9 & 5.3 & 2.0 & .7 & 1.1 \\
\hline & & & 1860 & 214 & 255 & 135 & 52 & 19 & 29 \\
\hline \multirow[t]{2}{*}{ Doing light gardening } & 2.25 & $.89(1.43)$ & 39.9 & 30.8 & 22.1 & 4.1 & 1.0 & 6 & .8 \\
\hline & & & 1024 & 790 & 568 & 106 & 26 & 16 & 21 \\
\hline \multirow[t]{2}{*}{ Doing heavy gardening } & 4.4 & $.92(1.73)$ & 56.2 & 16 & 17.9 & 5.5 & 2.3 & 6 & 1.4 \\
\hline & & & 1444 & 412 & 459 & 140 & 60 & 15 & 37 \\
\hline \multirow[t]{2}{*}{ Doing light housekeeping } & 2.5 & $3.17(3.42)$ & 21.5 & 15.4 & 24.1 & 11.6 & 6.6 & 5.1 & 14.6 \\
\hline & & & 552 & 396 & 620 & 298 & 170 & 130 & 376 \\
\hline \multirow[t]{2}{*}{ Doing heavy housekeeping } & 4.5 & $1.54(2.13$ & 33.1 & 22.0 & 27.1 & 9.9 & 3.2 & 1.8 & 2.6 \\
\hline & & & 849 & 565 & 697 & 255 & 82 & 46 & 67 \\
\hline \multirow[t]{2}{*}{ Jogging or running } & 7 & $.13(.58)$ & 91 & 4.3 & 3.6 & .8 & .1 & .1 & 0.0 \\
\hline & & & 2337 & 111 & 93 & 20 & 3 & 2 & 1 \\
\hline \multirow[t]{2}{*}{ Swimming } & 7 & $.11(.45)$ & 88.8 & 7.4 & 3.4 & .3 & 0,0 & 0,0 & 0.0 \\
\hline & & & 2280 & 190 & 87 & 8 & 1 & 0 & 1 \\
\hline \multirow[t]{2}{*}{ Playing (table) tennis, badminton } & 7 & $.17(.69)$ & 91.2 & 2.3 & 4.7 & 1.4 & .3 & .1 & 0.0 \\
\hline & & & 2342 & 58 & 121 & 36 & 6 & 3 & 0 \\
\hline \multirow[t]{2}{*}{ Playing a team sport indoors or outdoors } & 7.1 & $.11(.51)$ & 93.7 & 2 & 3.4 & .7 & .1 & .1 & 0.0 \\
\hline & & & 2406 & 52 & 87 & 18 & 2 & 2 & 0 \\
\hline \multirow{2}{*}{$\begin{array}{l}\text { Doing light exercise to maintain a physical } \\
\text { condition, e.g. stretching or flexibility exercises }\end{array}$} & 4 & $.33(.79)$ & 69.7 & 19.5 & 8.9 & 1.2 & .3 & .2 & .1 \\
\hline & & & 1790 & 501 & 229 & 30 & 7 & 4 & 2 \\
\hline \multirow{2}{*}{$\begin{array}{l}\text { Doing heavy exercises, e.g. aerobics, } \\
\text { fitness or strength training }\end{array}$} & 5 & $.27(.84)$ & 83.8 & 6.2 & 7.7 & 1.5 & .7 & .0 & .1 \\
\hline & & & 2152 & 159 & 197 & 39 & 17 & 1 & 2 \\
\hline
\end{tabular}

together, were less physically active. For a full overview of factors see Table 4 .

\section{Discussion}

This study was one of the first to investigate sociodemographic, medical and social-cognitive factors associated with PA behavior in large sample of individuals aged 45-70 years in the Netherlands. The present study showed that most popular PA activities among older adults appeared to be walking and cycling leisurely and household activities. According to the CHAMPS three-fourths of the respondents reported to perform at least two and a half hours of moderate physical activity a week. Previously we have shown that multiple item instrument as the Champs may overestimate PA level [42]. Therefore, it should be mentioned that the actual percentage might be lower. In this study $25 \%$ of the variance in PA was explained by age, gender, education, BMI, work situation, region of residence, smoking, awareness, advantages, descriptive norm, perceived behavioral control, habit, action plans and intention. Therefore, these variables were considered to be important to target in interventions aimed to increase PA in older adults. The later intervention study addressing these elements appeared successful in improving PA [25]. Although comparable studies on PA behavior measured with the CHAMPS questionnaire were not found, the present findings corroborated those of previous Theory of Reasoned action (TRA)/Theory of Planned Behavior (TPB) studies on physical activity regarding relevant correlates and percentages of variance explained. In a meta- 
Table 4 Linear regression of factors associated with physical activity

\begin{tabular}{|c|c|c|c|c|c|c|}
\hline \multirow[t]{2}{*}{ Variables } & \multicolumn{2}{|c|}{ Unstandardized coefficients } & \multirow[t]{2}{*}{$\mathbf{T}$} & \multirow[t]{2}{*}{ p-value } & \multicolumn{2}{|c|}{ Confidence interval } \\
\hline & $\beta$ & SE & & & Lower & Upper \\
\hline \multicolumn{7}{|l|}{ Step 1 Background factors* } \\
\hline Constant & 2.166 & .257 & 8.422 & .000 & 1.662 & 2.670 \\
\hline Age & -.014 & .003 & -4.411 & .000 & -.021 & -.008 \\
\hline \multicolumn{7}{|c|}{ Education level (low is reference category) } \\
\hline DUMMY intermediate education & .138 & .047 & 2.937 & .003 & .046 & .229 \\
\hline DUMMY high education & .167 & .048 & 3.460 & .001 & .072 & .262 \\
\hline BMI & -.021 & .004 & -4.975 & .000 & -.030 & -.013 \\
\hline Work situation & -.203 & .047 & -4.345 & .000 & -.294 & -.111 \\
\hline Gender & .166 & .039 & 4.219 & .000 & .089 & .243 \\
\hline Region of residence & .210 & .037 & 5.632 & .000 & .137 & .283 \\
\hline Smoking behavior & -.187 & .046 & -4.052 & .000 & -.278 & -.097 \\
\hline Awareness & -.233 & .038 & -6.176 & .000 & -.307 & -.159 \\
\hline Habit & .136 & .007 & 19.258 & .000 & .122 & .150 \\
\hline \multicolumn{7}{|l|}{ Step 2 Social cognitive factors** } \\
\hline Constant & 1.086 & .284 & 3.829 & .000 & .530 & 1.643 \\
\hline Age & -.010 & .003 & -3.156 & .002 & -.016 & -.004 \\
\hline \multicolumn{7}{|c|}{ Education level (low is reference category) } \\
\hline DUMMY intermediate education & .107 & .046 & 2.303 & .021 & .016 & .198 \\
\hline DUMMY high education & .144 & .048 & 3.008 & .003 & .050 & .237 \\
\hline BMl & -.019 & .004 & -4.474 & .000 & -.028 & -.011 \\
\hline Work situation & -.182 & .046 & -3.941 & .000 & -.273 & -.092 \\
\hline Gender & .195 & .039 & 5.009 & .000 & .119 & .271 \\
\hline Region of residence & .213 & .037 & 5.773 & .000 & .141 & .286 \\
\hline Smoking behavior & -.166 & .046 & -3.636 & .000 & -.256 & -.076 \\
\hline Awareness & -.207 & .037 & -5.512 & .000 & -.280 & -.133 \\
\hline Habit & .093 & .009 & 10.934 & .000 & .076 & .110 \\
\hline Advantages (attitude) & .007 & .002 & 3.065 & .002 & .003 & .012 \\
\hline Descriptive norm & -.016 & .007 & -2.163 & .031 & -.031 & -.002 \\
\hline Perceived behavioral control & .027 & .003 & 9.421 & .000 & .021 & .032 \\
\hline \multicolumn{7}{|c|}{ Step 3 Intention and actual control*** FINAL MODEL } \\
\hline Constant & 1.250 & .283 & 4.411 & .000 & .694 & 1.805 \\
\hline Age & -.011 & .003 & -3.470 & .001 & -.017 & -.005 \\
\hline \multicolumn{7}{|c|}{ Education level (low is reference category) } \\
\hline DUMMY intermediate education & .095 & .046 & 2.052 & .040 & .004 & .185 \\
\hline DUMMY high education & .135 & .048 & 2.834 & .005 & .042 & .228 \\
\hline BMI & -.019 & .004 & -4.408 & .000 & -.027 & -.010 \\
\hline Work situation & -.183 & .046 & -3.969 & .000 & -.273 & -.092 \\
\hline Gender & .191 & .039 & 4.947 & .000 & .115 & .267 \\
\hline Region of residence & .215 & .037 & 5.849 & .000 & .143 & .286 \\
\hline Smoking & -.165 & .045 & -3.644 & .000 & -.254 & -.076 \\
\hline Awareness & -.211 & .037 & -5.660 & .000 & -.284 & -.138 \\
\hline Habit & .083 & .009 & 9.481 & .000 & .066 & .100 \\
\hline Advantages (attitude) & .006 & .002 & 2.531 & .011 & .001 & .011 \\
\hline
\end{tabular}


Table 4 Linear regression of factors associated with physical activity (Continued)

\begin{tabular}{|c|c|c|c|c|c|c|}
\hline Descriptive norm & -.022 & .007 & -2.998 & .003 & -.037 & -.008 \\
\hline Perceived behavioral control & .019 & .003 & 6.038 & .000 & .013 & .025 \\
\hline Action plan & .039 & .018 & 2.248 & .025 & .005 & .074 \\
\hline Intention (stages) & .077 & .013 & 5.994 & .000 & .052 & .102 \\
\hline \multicolumn{7}{|l|}{ Women**** FINAL MODEL } \\
\hline Constant & 1.935 & .340 & 5.683 & .000 & 1.267 & 2.603 \\
\hline Age & -.018 & .004 & -4.330 & .000 & -.026 & -.010 \\
\hline BMI & -.016 & .006 & -2.822 & .005 & -.027 & -.005 \\
\hline Work situation & -.126 & .062 & -2.028 & .043 & -.248 & -.004 \\
\hline Region of residence & .257 & .051 & 4.998 & .000 & .156 & .358 \\
\hline Awareness & -.210 & .052 & -4.035 & .000 & -.313 & -.108 \\
\hline Habit & .058 & .012 & 4.643 & .000 & .012 & .030 \\
\hline Perceived behavioral control & .021 & .005 & 4.659 & .000 & .033 & .082 \\
\hline Action plan & .058 & .025 & 2.381 & .017 & .010 & .106 \\
\hline Intention (stage) & .085 & .019 & 4.571 & .000 & .048 & .121 \\
\hline \multicolumn{7}{|l|}{ Men $* * * * *$ FINAL MODEL } \\
\hline Constant & .946 & .260 & 3.637 & .000 & .436 & 1.457 \\
\hline \multicolumn{7}{|c|}{ Education level (low is reference category) } \\
\hline DUMMY intermediate education & .165 & .061 & 2.726 & .007 & .046 & .284 \\
\hline DUMMY high education & .217 & .062 & 3.498 & .000 & .095 & .338 \\
\hline BMI & -.024 & .006 & -3.784 & .000 & -.036 & -.011 \\
\hline Work situation & -.128 & .051 & -2.501 & .013 & -.229 & -.028 \\
\hline Alcohol consumption & -.171 & .069 & -2.486 & .013 & -.307 & -.036 \\
\hline Region of residence & .174 & .051 & 3.373 & .001 & .073 & .275 \\
\hline Marital status & -.168 & .069 & -2.429 & .015 & -.304 & -.032 \\
\hline Smoking & -.254 & .063 & -4.057 & .000 & -.377 & -.131 \\
\hline Awareness & -.197 & .052 & -3.798 & .000 & -.299 & -.095 \\
\hline Habit & .099 & .012 & 8.431 & .000 & .076 & .122 \\
\hline Perceived behavioral control & .019 & .004 & 4.654 & .000 & .011 & .028 \\
\hline Intention (stages) & .056 & .017 & 3.266 & .001 & .022 & .090 \\
\hline
\end{tabular}

Dependent variable: Hours/week moderately physically active; ${ }^{*}$ Step $1: R^{2}=.20$, ${ }^{* *}$ Step $2: R^{2}=.24,{ }^{* *}$ Step $3: R^{2}=.25 ;{ }^{* * * * W o m e n: ~ S t e p ~} 1: R^{2}=.18$, Step $2: R^{2}=.23$, Step 3: $\mathrm{R}^{2}=.24 ;{ }^{* * * *}$ Men: Step 1: $\mathrm{R}^{2}=.22$, Step 2: $\mathrm{R}^{2}=.25$, Step 3: $\mathrm{R}^{2}=.25$.

analytic review of 79 studies, Hagger et al. reported that the TRA accounted for $26 \%$ of the variance in PA behavior and the TPB explained $27 \%$ of the variance in PA behavior [12]. Similar percentages were also found in PA studies among older adults $[43,44]$.

Several of our background variables were not completely mediated by the TRA/TPB constructs indicating that there may be stable individual differences that influence PA. The shown influence of background factors may enrich our understanding of PA behaviour.

Even though many correlates were identified in the current study, $75 \%$ of PA variance remained unexplained. As stated, our percentages were similar to findings of other studies using diverse behavioral outcome measures, but a lack of correspondence between the social-cognitive measures in which we defined the investigated behavior as being moderately physically active for at least 30 minutes on five or more days a week and the CHAMPS measure (mapping specific behaviors) cannot be excluded [11].

To increase the explained variance, several predictors have been suggested as additions to the model as the contribution of environmental factors, diverse health status indicators, perceived health, (perceived) lack of leisure time, mood disturbance and perceived PA effort [45]. In this study three health status indicators, diabetes, hypertension and BMI were added to the predictor list. Only BMI seemed to have an effect on PA. The observed negative influence of BMI on PA levels was in line with previous studies among the same age group $[18,43]$. Gauvin et al. noted that women with higher BMIs experience PA 
not as pleasurable and feel embarrassment when seen in public with exercise clothes [43]. However, this explanation did not hold for men and requires more research. In future interventions this health indicator should get extra attention.

The decline in PA levels with age is consistent with other studies conducted among a similar age group $[18,46]$. Norman et al. stated that poorer health or perceived lack of good health may induce that older people engage less often in PA [18]. Dergance et al. believed that fear of injuries may contribute to the decline in PA level among older individuals [47]. Additionally, Berger et al. suggested that unfavorable cultural expectations and norms exist about PA among older adults [46]. The general perception is that social norms demand that people relax and that more vigorous PA is not an appropriate behavior for older adults [43]. Unfortunately, social norm was not measured in the current study to support this assumption since it rarely was a significant predictor in previous studies on PA [44]. Another reason for the decline in PA levels by age is given by Norman and colleagues, who claimed that the lower PA level of aging people was associated with a lower level of occupation and occupational activities among older adults [18].

In contrast to other studies among the same age group $[18,46]$, the current findings indicate that women older than 45 years were more physically active than men of same age. This was also found in a Scottish sample among individuals aged 75 or older [48]. The increased activity among older women could be explained due to extra available time caused by a decrease in workload at home and care-giving for children, who live now outside the home [49]. Another reason could be that men achieve their PA levels by playing sports (e.g. football), but with increasing age these activities become harder to continue [50].

Besides the current study, several researchers found that employees older than 45 years are less physically active e,g, [18]. One explanation is that employees may be physically active at their work place or a have a physically active job, an aspect that is often not taken into account in PA questionnaires. Moreover, Berger at al. observed that the more physically demanding work is, the less likely people are to engage in PA outside work, meaning PA at work compensates PA in leisure times [46]. However, the current study cannot support this argument as PA at work was not measured.

Consistent with the findings reported elsewhere [18,48], the current results confirmed that high educated older individuals have higher PA levels. An explanation is that high educated individuals have a better health consciousness and more knowledge about advantages than lower educated individuals. There are also indications that high educated individuals have a higher perceived behavioral control and perceive fewer barriers engaging in PA [51]. In the current study, a t-test analysis was conducted comparing high versus low educated, which partly supported the assumptions. Compared to low educated, perceived behavioral control was higher among high educated but non-significantly. In addition, high educated individuals experienced significantly fewer disadvantages (barriers) but also fewer advantages compared to low educated.

Smokers appeared less physically active than nonsmokers. This was also found by others $[18,46]$. The finding can be explained by the fact that health risk behaviors cluster, and that smoking has a disruptive effect (e.g. decreased lung function) on PA performance [52].

Additionally, the results of this study suggest that men are less physically active when drinking alcohol above the national norm. The few studies that investigated the relationship between alcohol consumption and PA found no significant association [50,53]. To our knowledge no study exists regarding the influence of alcohol on PA behavior among older adults and possible gender differences.

The present data, and those of others [54], identified individuals living in North Limburg and North-Brabant as being more physically active than individuals in South Limburg. Mulder reported that South Limburg with 46.8\% has the lowest number of individuals older than 12 years meeting the national physical activity norm [54]. South Limburg, in contrast to the North, is a hilly area which might cause the lower level of PA. Several studies considered hilly terrains as barrier for PA [55].

With respect to the social-cognitive factors, the current study has demonstrated that individuals, who were not aware of and overestimated their own PA levels, were less physically active. Other studies conducted among Dutch adults (18 and older) confirmed this finding [56,57]. Ronda et al. concluded that $61.1 \%$ of the individuals with inadequate PA levels overestimate their PA level and thus making the motivation to increase PA in these groups difficult [40].

The positive influence of perceived behavioral control on PA found in the present study is in line with previous studies among the similar age group [58,59]. Perceived behavioral control is acknowledged as the most important psychological factor for PA e.g. [12,45]. High perceived behavioral control allows people to set reachable goals and overcome barriers, which enable them to be physically active [60]. Rovniak et al. [61] found in university students that perceived behavioral control was mediated by self-regulation strategies such as goal setting, self-monitoring, planning and problem solving. Moreover, personal achievement to be physically active, experiencing other people accomplishing engagement in PA (social comparison) or verbal persuasion by others to participate in PA increase perceived behavioral control [63]. In the current study, individuals observing other persons being physically active or having social support 
also had significantly higher perceived behavioral control, as a t-test assessment revealed.

Several studies demonstrated that action plans are important to overcome the gap between intention and actual behavior [62-64]. This means, specifying PA behavior using parameters such as 'when', 'where' and 'how', leads to actual PA behavior. According to Reuter et al., older adults are more successful in implementing their plans despite barriers due to more experienced self-regulation mechanisms (e.g. goal-setting) [62]. However, our study did not confirm that adults who are older have more action plans.

Consistent with the findings reported in other studies among broader age groups [e.g. 12], the present study showed that habit strength was associated with PA behavior. Behaviors have been once initiated by rational choices and later they have been formed to habits, which are triggered by cues without cognitive processes [65]. Additionally, positive experiences (e.g. emotions and enjoyment) of PA are important for the formation of habits [65]. Positive feelings during PA increase positive attitude [65]. Keviniemi et al. found that positive affective associations can serve as cognitive shortcuts to PA [66]. This means that individuals decide to engage in PA without using a rational decision-making process each time, but because they expect, for example, enjoyment from the PA. In line with other studies [67], the finding of the present study also support that individuals believing more strongly in the health benefits of PA are more physically active.

In the current study, participants were not motivated to engage in PA by observing other physically active people in their environment. This is in contrast to most studies among similar age groups $[67,68]$. However, Wilcox and colleagues studied sedentary behavior among women 40 years and older. They also found that women who see others exercise, have a more sedentary behavior [69]. Moreover, a study among women 20 to 50 years old demonstrated that female urban Latinas and urban African Americans knowing people who exercise were less physically active [70]. Unfortunately, neither study supported their finding with an explanation. Reasons might be that they do not perceive others as role models for PA so that they cannot identify themselves with others.

Consistent with the findings reported elsewhere [17,71], older individuals with the intention to take part in PA are also more physically active. According to Fishbein \& Ajzen, forming the intention to engage in PA is translated into actual PA behavior [11].

\section{Study strength and limitations}

The current study had several strengths including the origin of the study population, the large sample size, the random selection from different GP practices, the inclusion of medical characteristics (e.g. hypertension), and the generalizability of results with regard to gender and education level. Moreover, it is one of the first studies concentrating on factors influencing PA in older individuals.

Limitations of the study are the cross-sectional design and the measurement of PA behavior through a selfreported questionnaire. Self-report measurements require from participants good memories and estimation skills. Consequently, measurement errors may exist due to social desirable answers or lack of valid recall [72]. Moreover, the PA measure lacks detail and specificity because it focuses on common activities and misses workplace physical activity. Thus, PA behavior may be over- or underestimated [73]. Another limitation of the study is the use of a long questionnaire to measure PA and the other variables [74], which may result in nonresponse and invalid results [75]. In the Netherlands about one third of the population (aged 30-70 years) suffers from hypertension [76]. For research purposes the number of people who have hypertension was higher (50\%) than normal. Nevertheless, hypertensive people did not seem to be more physically active than normotensive people in this study. Finally, the study was limited due to the lack of relatively more objective measures such as activity monitors to validate the selfreport questionnaires, because the use of these objective measures was considered too time-consuming and expensive in such a large population [77].

\section{Conclusion}

The current study adds to the literature by identifying important socio-demographic, medical and social-cognitive associates of PA in individuals aged $45-70$ years. Consequently, it contributes to the ability to develop PA interventions tailored to older adults. The following correlates of PA should be considered in the development of these interventions: age, gender, education, BMI, work situation, region of residence, smoking, awareness, advantages (attitude), descriptive norm, perceived behavioral control, habit, action plans and intention. By considering these factors in regional or national PA interventions, a higher level of PA among older adults could be stimulated and as a result the public health burden may be minimized through a decrease in CVDs among this large population group.

\section{Abbreviation}

BMI: Body mass index; CVDs: Cardiovascular diseases; CHAMPS: Community healthy activities model program for seniors; PA: Physical activity; TPB: Theory of planned behavior; TRA: Theory of reasoned action.

\section{Competing interest}

The authors declare that they have no competing interests.

\section{Authors' contribution}

HMVK carried out the Vitalum Study, helped with the statistical analysis, and revised the manuscript critically. IM helped to draft the manuscript and to interpret the data, and revised it critically. SW performed the statistical analysis, did the interpretation of the data and drafted the manuscript. All authors have seen and approved of the version to be published. 


\section{Acknowledgement}

We would like to thank the participants for taking part in Vitalum, the GPs in the recruitment of participants, the MEMIC centre for data entry and information management.

\section{Funding}

This work was supported by The Netherlands Organization for Health Research and Development [ZonMw, grant number 22000120]. It was conducted under the auspices of the School for Public Health and Primary Care (www.caphri.nl)

\section{Author details}

'School for Public Health and Primary Care (Caphri), Department of Epidemiology, Maastricht University, PO Box 616, Maastricht 6200 MD, The Netherlands. ${ }^{2}$ TNO, Expertise Center Life Style, P.O. Box 2215, Leiden 2301 CE, The Netherlands.

Received: 8 November 2013 Accepted: 19 June 2014

Published: 25 June 2014

\section{References}

1. Cardiovascular diseases (CVDs) Fact sheet $\mathrm{N}^{\circ} 317$. http://www.who.int/ mediacentre/factsheets/fs317/en/index.html.

2. Sofi F, Capalbo A, Cesari F, Abbate R, Gensini GF: Physical activity during leisure time and primary prevention of coronary heart disease: an updated meta-analysis of cohort studies. Eur J Cardiovasc Prev Rehabil 2008, 15:247-257.

3. Nocon M, Hiemann T, Müller-Riemenschneider F, Thalau F, Roll S, Willich SN: Association of physical activity with all-cause and cardiovascular mortality: a systematic review and meta-analysis. Eur J Cardiovasc Prev Rehabil 2008, 15:239-246.

4. Li J, Siegrist J: Physical activity and risk of cardiovascular disease-a meta-analysis of prospective cohort studies. Int J Environ Res Public Health 2012, 9:391-407

5. Haskell WL, Lee I, Pate RR, Powell KE, Blair SN, Franklin BA, Macera CA, Heath GW, Thompson PD, Bauman A: Physical activity and public health: updated recommendation for adults from the American College of Sports Medicine and the American Heart Association. Med Sci Sports Exerc 2007, 39:1423-1434.

6. Warburton DE, Nicol CW, Bredin SS: Health benefits of physical activity: the evidence. Can Med Assoc J 2006, 174:801-809.

7. Garber C, Blissmer B, Deschenes M, Franklin B, Lamonte M, Lee I, Nieman D, Swain D: American College of Sports Medicine position stand. Quantity and quality of exercise for developing and maintaining cardiorespiratory, musculoskeletal, and neuromotor fitness in apparently healthy adults: guidance for prescribing exercise. Med Sci Sports Exerc 2011, 43:1334-1359.

8. Centraal Bureau voor de Statistiek: Lichamelijke activiteit (12+) Voldoen aan norm gezond bewegen. In StatLine; 2011. https://www.tno.nl/ downloads/trendrapport_bewegen_gezondheid_2010_2011.pdf

9. Green LW, Kreuter MW: Health program planning: an educational and ecological approach. In New York, NY: McGraw-Hill; 2005

10. Bartholomew LK, Parcel GS, Kok G, Gottlieb NH, Fernandez ME: Planning health promotion programs: an intervention mapping approach. In San Frascisco: Jossey-Bass; 2011

11. Fishbein M, Aizen I: Predicting and changing behavior: the reasoned action approach. In New York: Psychology Press; 2009.

12. Hagger MS, Chatzisarantis NL, Biddle SJ: A meta-analytic review of the theories of reasoned action and planned behavior in physical activity: predictive validity and the contribution of additional variables. J Sport Exerc Psychol 2002, 24:3-32.

13. Vries HD, Backbier E, Kok G, Dijkstra M: The impact of social influences in the context of attitude, self efficacy, intention, and previous behavior as predictors of smoking onset. J App/ Soc Psychol 1995, 25:237-257.

14. Anderson ES, Wojcik JR, Winett RA, Williams DM: Social-cognitive determinants of physical activity: the influence of social support, selfefficacy, outcome expectations, and self-regulation among participants in a church-based health promotion study. Health Psychol 2006, 25:510-520.

15. Pan $\mathrm{S}$, Cameron $\mathrm{C}$, DesMeules $M$, Morrison H, Craig C, Jiang $X$ : Individual, social, environmental, and physical environmental correlates with physical activity among Canadians: a cross-sectional study. BMC Public Health $2009,9: 21$.
16. Sassen B, Kok G, Schaalma H, Kiers $H$, Vanhees $L$ : Cardiovascular risk profile: cross-sectional analysis of motivational determinants, physical fitness and physical activity. BMC Public Health 2010, 10:592.

17. Caudroit J, Stephan Y, Le Scanff C: Social cognitive determinants of physical activity among retired older individuals: An application of the health action process approach. Br J Health Psychol 2011, 16:404-417.

18. Norman A, Bellocco R, Vaida F, Wolk A: Total physical activity in relation to age, body mass, health and other factors in a cohort of Swedish men. Int J Obes Relat Metab Disord 2002, 26:670-675.

19. Eurostat: People by age classes. Share of total population (\%). 2011, Retrieved Mai 28, 2014, from the world wide web: http://epp.eurostat.ec. europa.eu/.

20. Shiroma EJ, Lee IM: Physical activity and cardiovascular health lessons learned from epidemiological studies across age, gender, and race/ ethnicity. Circulation 2010, 122:743-752.

21. Metsemakers JF, Höppener P, Knottnerus JA, Kocken RJ, Limonard CB: Computerized health information in The Netherlands: a registration network of family practices. Br J Gen Pract 1992, 42:102-106.

22. Lamberts $\mathrm{H}$, Wood M: Party I: ICPC, international classification of primary care. USA: Oxford University Press; 1988.

23. Nederlands Huisartsen Genootschap: NHG-standaard cardiovasculair risicomanagement. In Houten: Bohn Stafleu van Loghum; 2006.

24. van Keulen HM, Mesters I, Brug J, Ausems M, Campbell M, Resnicow K, Zwietering PJ, Van Breukelen G, Van Mechelen W, Severens JL: Vitalum study design: RCT evaluating the efficacy of tailored print communication and telephone motivational interviewing on multiple health behaviors. BMC Public Health 2008, 8:216.

25. van Keulen HM, Mesters I, Ausems M, van Breukelen G, Campbell M, Resnicow K, Brug J, de Vries H: Tailored print communication and telephone motivational interviewing are equally successful in improving multiple lifestyle behaviors in a randomized controlled trial. Ann Behav Med 2011, 41:104-118.

26. Prochaska JO, DiClemente CC, Norcross JC: In search of how people change: applications to addictive behaviors. Am Psychol 1992, 47:1102-1114.

27. Brug J, Van Assema P, Kok G, Lenderink T, Glanz K: Self-rated dietary fat intake: association with objective assessment of fat, psychosocial factors, and intention to change. J Nutr Educ 1994, 26:218-218.

28. Corbin CB, Welk GJ, Corbin WR, Welk KA: Concepts of physical fitness. Active lifestyles for wellness. In New York: McGraw-Hill; 2004.

29. Mudde A: Meetinstrumenten voor onderzoek naar roken en stoppen met roken [measurements for research about smoking and stop smoking]. In Den Haag: Stivoro; 2000.

30. Lemmens $P, \operatorname{Tan} E$, Knibbe R: Measuring quantity and frequency of drinking in a general population survey: a comparison of five indices. J Stud Alcohol Drugs 1992, 53:476-486.

31. Gezondheidsraad: Richtlijnen goede voeding 2006 - achtergronddocument. In Den Haag: Gezondheidsraad; 2006.

32. Atkinson G, Drust B: Seasonal rhythms and exercise. Clin Sports Med 2005 24:e25-e34.

33. Resnicow K, McCarty F, Blissett D, Wang T, Heitzler C, Lee RE: Validity of a modified CHAMPS Physical Activity Questionnaire among AfricanAmericans. Med Sci Sports Exerc 2003, 35:1537-1545.

34. Stewart AL, Mills KM, King AC, Haskall WL, Gillis D, Ritter PL: CHAMPS physical activity questionnairew for older adults: outcomes for intervention. Med Sci Sports Exerc 2001, 33:1126-1141.

35. Ainsworth BE, Haskell WL, Whitt MC, Irwin ML, Swartz AM, Strath SJ, O'Brien WL, Bassett DR Jr, Schmitz KH, Emplaincourt PO, Jacobs DR Jr, Leon AS: Compendium of physical activities: an update of activity codes and MET intensities. Med Sci Sports Exerc 2000, 32:S498-S504.

36. Pate RR, Pratt M, Blair SN, Haskell WL, Macera CA, Bouchard C, Buchner D, Ettinger W, Heath GW, King AC, Krisk A, Leon AS, Marcus BH, Morris J, Paffenbarger R, Patrick K, Pollock ML, Rippe JM, Sallis J, Wilmore JH: Physical activity and public health. A recommendation from the centers for disease control and Prevention and the American College of Sports Medicine. JAMA 1995, 273:402-407.

37. Harada ND, Chiu V, King AC, Stewart AL: An evaluation of three self-report physical activity instruments for older adults. Med Sci Sports Exerc 2001 33:962-970

38. Pruitt LA, Glynn NW, King AC, Guralnik JM, Aiken EK, Miller G, Haskell WL: Use of accelerometry to measure physical activity in older adults at risk for mobility disability. J Aging Phys Act 2008, 16:416-434 
39. Giles K, Marshall AL: The repeatability and accuracy of CHAMPS as a measure of physical activity in a community sample of older Australian adults. J Phys Act Health 2009, 6:221-229.

40. Ronda G, Van Assema P, Brug J: Stages of change, psychological factors and awareness of physical activity levels in the Netherlands. Health Promot Int 2001, 16:305-314.

41. Tabachnick B, Fidell L: Using multivariate statistics. In Needham Heights, United Kingdom: Pearson Higher Education; 2006.

42. van Keulen HM, Mesters I, van Mechelen W, de Vries H: Single-item and multiple-itemmeasures of adherence to public health behavior guidelines were incongruent. J Clin Epidemiol 2010, 63:75-84

43. Gretebeck KA, Black DR, Blue CL, Glickman LT, Huston SA, Gretebeck RJ: Physical activity and function in older adults: theory of planned behavior. Am J Health Behav 2007, 31:203-214.

44. Plotnikoff RC, Lippke S, Courneya K, Birkett N, Sigal R: Physical activity and diabetes: an application of the theory of planned behaviour to explain physical activity for Type 1 and Type 2 diabetes in an adult population sample. Psychol Health 2010, 25:7-23.

45. Trost SG, Owen N, Bauman AE, Sallis JF, Brown W: Correlates of adults' participation in physical activity: review and update. Med Sci Sports Exerc 2002, 3:1996-2001.

46. Berger U, Der G, Mutrie N, Hannah MK: The impact of retirement on physical activity. Aging Ment Health 2005, 25:181-195.

47. Dergance JM, Calmbach WL, Dhanda R, Miles TP, Hazuda HP, Mouton CP: Barriers to and benefits of leisure time physical activity in the elderly: differences across cultures. J Am Geriatr Soc 2003, 51:863-868.

48. Murray L: Exercise and physical activity: public participation, barriers and attitudes. In Edinburgh: Scottish Executive Education Department; 2006.

49. Physical activity and women. http://www.who.int/dietphysicalactivity/ factsheet women/en/.

50. Sallis JF, Richard Hofstetter C, Faucher P, Elder JP, Blanchard J, Caspersen CJ, Powell KE, Christenson GM: A multivariate study of determinants of vigorous exercise in a community sample. Am J Prev Med 1989, 18:20-34

51. Rhodes RE, Martin AD, Taunton JE, Rhodes EC, Donnelly M, Elliot J: Factors associated with exercise adherence among older adults: an individual perspective. Sports Med 1999, 28:397-411.

52. Sherwood NE, Jeffery RW: The behavioral determinants of exercise: implications for physical activity interventions. Annu Rev Nutr 2000, 20:21-44.

53. Blair SN, Jacobs DR Jr, Powell KE: Relationships between exercise or physical activity and other health behaviors. Public Health Rep 1985, 100:172-180.

54. Mulder M: Norm gezond bewegen 2005-2008. In Volksgezondheid Toekomst Verkenning, Nationale Atlas Volksgezondheid. Bilthoven: RIVM; 2010.

55. Sallis JF, Bowles HR, Bauman A, Ainsworth BE, Bull FC, Craig CL, Sjöström M, De Bourdeaudhuij I, Lefevre J, Matsudo V, Matsudo S, Macfarlane DJ, Gomez LF, Inoue S, Murase N, Volbekiene V, McLean G, Carr H, Heggebo LK, Tomten H, Bergman P: Neighborhood environments and physical activity among adults in 11 countries. Am J Prev Med 2009, 3:6484-6490.

56. Lechner L, Bolman C, Van Dijke M: Factors related to misperception of physical activity in The Netherlands and implications for health promotion programmes. Health Promot Int 2006, 21:104-112.

57. van Sluijs E, Griffin S, van Poppel M: A cross-sectional study of awareness of physical activity: associations with personal, behavioral and psychosocial factors. Int J Behav Nutr Phys Act 2007, 4:53.

58. Booth ML, Owen N, Bauman A, Clavisi O, Leslie E: Social-cognitive and perceived environment influences associated with physical activity in older Australians. Am J Prev Med 2000, 31:15-22

59. Burton LC, Shapiro S, German PS: Determinants of physical activity initiation and maintenance among community-dwelling older persons. Am J Prev Med 1999, 29:422-430.

60. Thirlaway K, Upton D: The Psychology of Lifestyle: promoting healthy behaviour. London and New York: Routledge; 2009.

61. Rovniak LS, Anderson ES, Winett RA, Stephens RS: Social cognitive determinants of physical activity in young adults: a prospective structural equation analysis. Ann Behav Med 2002, 24:149-156.

62. Reuter T, Ziegelmann JP, Wiedemann AU, Lippke S, Schüz B, Aiken LS: Planning bridges the intention-behaviour gap: age makes a difference and strategy use explains why. Psychol Health 2010, 25:873-887.

63. Scholz U, Sniehotta FF, Burkert S, Schwarzer R: Increasing physical exercise levels age-specific benefits of planning. J Aging Health 2007, 19:851-866.
64. Ziegelmann JP, Lippke S, Schwarzer R: Adoption and maintenance of physical activity: planning interventions in young, middle-aged, and older adults. Psychol Health 2006, 21:145-163.

65. Aarts H, Paulussen T, Schaalma H: Physical exercise habit: on the conceptualization and formation of habitual health behaviours. Health Educ Res 1997, 12(3):363-374.

66. Kiviniemi MT, Voss-Humke AM, Seifert AL: How do i feel about the behavior? The interplay of affective associations with behaviors and cognitive beliefs as influences on physical activity behavior. Health Psychol 2007, 26:152-158.

67. Booth KM, Pinkston MM, Poston WSC: Obesity and the built environment J Am Diet Assoc 2005, 105:110-117.

68. King AC, Castro C, Wilcox S, Eyler AA, Sallis JF, Brownson RC: Personal and environmental factors associated with physical inactivity among different racial-ethnic groups of US middle-aged and older-aged women. Health Psychol 2000, 19:354-364.

69. Wilcox S, Castro C, King AC, Housemann R, Brownson RC: Determinants of leisure time physical activity in rural compared with urban older and ethnically diverse women in the United States. J Epidemiol Community Health 2000, 54:667-672

70. Eyler AA, Matson-Koffman D, Young DR, Wilcox S, Wilbur J, Thompson JL, Sanderson B, Evenson KR: Quantitative study of correlates of physical activity in women from diverse racial/ethnic groups: the women's cardiovascular health network project summary and conclusions. Am J Prev Med 2003, 25:93-103.

71. Renner B, Spivak Y, Kwon S, Schwarzer R: Does age make a difference? Predicting physical activity of South Koreans. Psychol Aging 2007, 22:482-493.

72. Matthews CE: Use of self-report instruments to assess physical activity. In Physical activity assessments for health-related research. Edited by Welk G. Champaign: Human Kinetics; 2002:107-123.

73. Schwarz N: Self-reports: how the questions shape the answers. Am Psychol 1999, 54:93-105.

74. Brug J, Oenema A, Campbell M: Past, present, and future of computertailored nutrition education. Am J Clin Nutr 2003, 77:1028S-1034S.

75. Kremers SP, Visscher TL, Seidell JC, Van Mechelen W, Brug J: Cognitive determinants of energy balance-related behaviours: measurement issues. Sports Med 2005, 35:923-933.

76. Blokstra A, VanBakel AM: Hoeveel mensen hebben een erhoogde bloeddruk? In Volksgezondheid Toekomst Verkenning, Nationaal Kompas Volksgezondheid. Bilthoven: RIVM; 2012. http://www.nationaalkompas.nl> Nationaal Kompas Volksgezondheid\Determinanten\Persoonsgebonden\Bloeddruk.

77. Welk G: Physical activity assessments for health-related research. Champaign: Human Kinetics; 2002.

doi:10.1186/1471-2458-14-647

Cite this article as: Mesters et al:: Socio-demographic, medical and social-cognitive correlates of physical activity behavior among older adults (45-70 years): a cross-sectional study. BMC Public Health $201414: 647$

\section{Submit your next manuscript to BioMed Central and take full advantage of:}

- Convenient online submission

- Thorough peer review

- No space constraints or color figure charges

- Immediate publication on acceptance

- Inclusion in PubMed, CAS, Scopus and Google Scholar

- Research which is freely available for redistribution 